\title{
Complications associated with Mitchell's Osteotomy for Hallux Valgus Correction: A retrospective hospital review
}

\author{
by Kenneth. S. David-West, FRCS (Ed), FICS, FWACS (Orth. \& Tr.) ${ }^{1 凹}$
}

The Foot and Ankle Online Journal 4 (3): 1

Background: A retrospective review of complications in Mitchell's osteotomy for mild to moderate hallux valgus correction, in which all osteotomies were fixed with sutures.

Methods: The study evaluated Mitchell's osteotomy from a district General hospital. There were 82 feet in 68 patients (14 patients had bilateral osteotomies). The study included 60 females and 8 males; the mean age being 54 years, and the average follow-up was 9 months (6-24 months).

Results: Thirty seven feet (45.0\%) had complications, of which 22 feet had recurrence and of these, 9 feet had recurrence plus transfer metatarsalgia, One foot had avascular necrosis, 2 feet with non union, one foot with cock-up deformity and two with infection. In the feet with recurrence (22 feet) there was no correction of the tibial sesamoid position.

Conclusions: This study demonstrates that Mitchell's osteotomy for hallux valgus correction fixed with sutures is associated with high recurrence and transfer metatarsalgia.

Key words: Mitchell's bunionectomy, Mitchell's osteotomy, bunion complications.

This is an Open Access article distributed under the terms of the Creative Commons Attribution License. It permits unrestricted use, distribution, and reproduction in any medium, provided the original work is properly cited. (The Foot and Ankle Online Journal (www.faoj.org), 2011 All rights reserved.

L eslie Mitchell in 1958 described an osteotomy of the distal first metatarsal for the correction forefoot deformity which affects females more than males. There are over 150 different procedures for hallux valgus corrections which include soft tissue and bone operations. $^{2}$

Address correspondence to: Mr. K .S. David-West, 14 Kirkaig Avenue, Dean Park, Renfrew, PA4 0YH. Scotland.

Tel: +441415612103 or +447887766283

${ }^{1}$ Orthopaedic Department, Crosshouse Hospital, Kilmarnock, KA2 0BE, Scotland, UK.
Mild deformities are usually corrected with a distal osteotomy, while moderate and severe deformities are corrected with diaphyseal or proximal osteotomies. ${ }^{3,4}$

Despite several techniques for hallux valgus correction, there is much controversy regarding the best procedure to use in a severe deformity, ${ }^{5}$ Mitchell and Scarf osteotomies do not restore the load bearing function of the foot to normal, ${ }^{6}$ Mitchell's osteotomy appears to be performed in a variety of different ways $^{7}$, some modifications, such as the planter displacement of the metatarsal head has been shown to be important. ${ }^{8}$ 


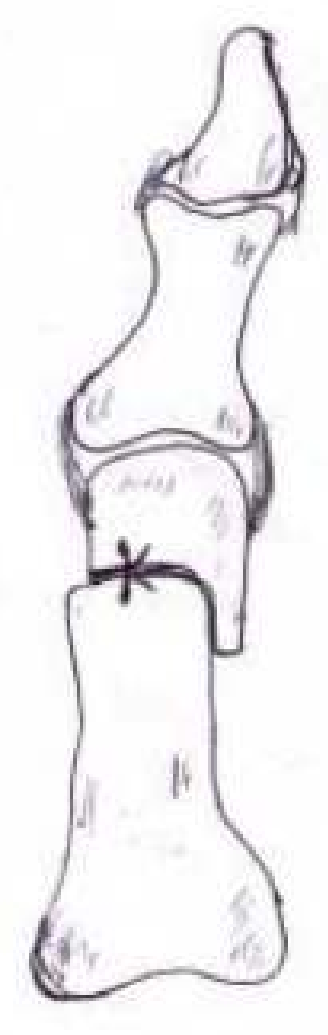

Figure 1 Mitchell's osteotomy fixed with suture.

Mitchell's osteotomy has been used widely in the correction of hallux valgus deformities and is known to be associated with complications.

Some of the reported complications include transfer metatarsalgia, mal union, recurrence, avascular necrosis and non-union. Good outcomes have been reported with Modified Mitchell's osteotomy in which the intermetatarsal angle is less than 20 degrees, offering a stable construct with easy post-operative care. ${ }^{5}$ Mitchell' osteotomies are associated with $40 \%$ of transfer metatarsalgia.

This is a retrospective review of the complications of Mitchell's osteotomies that were stabilized with sutures with an attempt to identify the incidence of recurrence and transfer metatarsalgia.

\section{Methods}

A retrospective review of Mitchell's osteotomy from a district General hospital performed between January 2007 and December 2009 was studied. The patients were identified from the hospital coding databank. 82 feet in 68 patients were studied. Fourteen patients had bilateral Mitchell's osteotomies. Patients' records and radiographs were reviewed retrospectively noting the indication for surgery, method of osteotomy fixation and recurrence, shortening, avascular necrosis, infections and the positions of the sesamoid pre and post operation and the experience of the surgeons. Their radiographs at pre-operation, 2 weeks, 6 weeks and latest follow up were reviewed from the patient archive communication system (PAC system). The average follow-up was 9 months (range 6- 24 months).

The criteria for recurrence is pain over the bunion with clinical recurrence of deformity and loss of correction of the hallux valgus angle (HVA) $\geq 18^{\circ}$ and intermetatarsal angle (IMA) $\geq 13^{\circ}$ in the last follow-up weight bearing $\mathrm{x}$-ray. The criteria for transfer metatarsalgia is a patient complaining of pain over the lesser toe metatarsal head and tenderness on clinical examination. All the surgeries were performed by a consultant or registrar. There was no significant difference in the complication noted between the surgeons.

\section{Surgical Technique}

All the patients had classical Mitchell's osteotomy. This is a distal "Step Cut" metatarsal osteotomy that displaces the metatarsal head laterally in order to correct hallux valgus deformity. A dorsomedial incision is carried down to the joint capsule; a dorsal and plantar skin flap is created, with care being taken to avoid the cutaneous nerves.

A distally based flap is then created from the medial joint capsule in order to expose the medial eminence and is removed in line with the medial aspect of the metatarsal shaft. 
Table 1 Complications (in 37 feet $45 \%$ of 82 feet total reviewed)

\begin{tabular}{lcl}
\hline Complications & $\begin{array}{c}\text { Number } \\
\text { Of complications }\end{array}$ & $\begin{array}{l}\% \text { of } 82 \text { feet } \\
\text { (total reviewed) }\end{array}$ \\
\hline Recurrences & 22 & $26.8 \%$ \\
Transfer metatarsalgia & 9 & $10.9 \%$ \\
Infection & 2 & $2.4 \%$ \\
Avascular necrosis & 1 & $1.2 \%$ \\
Non-union & 2 & $2.4 \%$ \\
Cock -up deformity & 1 & $1.2 \%$ \\
\hline
\end{tabular}

Total $37 \quad 45 \%$ of 82 feet

Transverse osteotomy is performed through the metatarsal neck, just proximal to the level of sesamoids between 2 cortical holes. However, a lateral $3 \mathrm{~mm}$ of metatarsal cortex is left uncut. The lateral cortex is then cut proximal to the first cut which creates a step off in the distal fragment. The distal fragment is then displaced laterally by the width of step off and is placed into a slight degree of plantar flexion to accommodate the degree of shortening that had been created by removing the section of bone. The greater the deformity the larger was the need for more lateral displacement. The osteotomy site is then secured with an Ethibond ${ }^{\mathrm{TM}}$ suture. The medial joint capsule is plicated in order to align MP joint. (Fig. 1)

Shortening of the first metatarsal and transfer metatarsalgia to the second metatarsal is compensated by plantar flexion of the distal fragment at the time of surgery. A post operative toe spike cast is applied and allowed to bearing weight on the heels with post operation shoes. Stitches are removed in two week and, an x-ray taken, and then a plaster slipper cast is applied for another 4 weeks after which another x-ray is taken at 6 weeks. The average hospital stay was 36 hours.

\section{Results}

From January 2007 to December 2009, 82 feet in 68 patients that had Mitchell's osteotomy for hallux valgus were reviewed that included 60 females and 8 males. Four had bilateral procedures. The mean age of the patients was 54 years (range 20-68 years).

Of the 82 feet reviewed 37 had complications (45.0\%). Of which 22 feet $26.8 \%$ had recurrence, 9 feet $10.9 \%$ had transfer metatarsalgia. 2 feet $(2.4 \%)$ had an infection, one superficial and one deep infection managed with debridement and antibiotics. (Table 1) 2 feet had non union these were diagnosed by pain at the osteotomy site and radiological signs of non union at 9 month's x-ray, and 1 foot had cock-up deformity treated with Z-plasty of extensor hallucis longus (EHL). One foot had avascular necrosis 1.2\% (Table 1) In feet which had recurrence, the intermetatarsal angle (IMA) of the patients were high, the mean IMA pre-operatively was $18.8^{\circ}$ and the hallux valgus (HA) angle was $34.5,^{\circ}$ with a mean distal metatarsal articular angle (DMAA) of 11.8 degrees. (Table 2) 
Table 2 Radiographic Measurements of feet with recurrence (mean. $\pm S D, N=22$ )

\begin{tabular}{lll}
\hline & Preoperative & Latest Follow-up \\
\hline Hallux valgus angle (HVA) & $34.5^{\circ} \pm 3.8^{\circ}$ & $36.4^{\circ} \pm 4^{\circ}$ \\
Intermetatarsal angle (IMA) & $18.8^{\circ} \pm 4.2^{\circ}$ & $18.2^{\circ} \pm 3.6^{\circ}$ \\
\hline Distal metatarsal articular angle & $11.8^{\circ} \pm 3.1^{\circ}$ & $11^{\circ} \pm 2.4^{\circ}$ \\
\hline Tibial Sesamoid (Grade 2) & 14 & 16 \\
(Grade 3) & 8 & 6 \\
\hline
\end{tabular}

Review of the tibial sesamoid in feet with recurrence showed that 14 feet are grade 2 and 8 feet are grade 3 pre-operatively and post-operatively 16 feet are grade 2 and 6 feet are grade 3. (Table. 2) These positions are considered 'abnormal'. ${ }^{10}$ None of the feet with recurrence had tibial sesamoid position corrected; hence there is not significant change in the position of the tibial sesamoid. There was no immediate post radiographic to show if the tibial sesamoid were corrected at time of the operation.

Of the 9 feet with transfer metatarsalgia, 5 had additional surgery to the lesser toes, see (Table 3), including partial proximal phalangectomy to 4 feet and excision of proximal interphalangeal joint in 1 foot. The procedures were performed at same time as the Mitchell's osteotomy.

\section{Discussion}

Mitchell's osteotomy is one of the distal metatarsal osteotomies used in the correction of hallux valgus, but the complications associated with the procedure make it less attractive.

Some modifications such as the planter displacement of the metatarsal head have been shown to be important ${ }^{8}$, but many modifications are not supported by scientific evidence. In addition a number of surgeons have not altered their practice in accordance with published evidence. In the Northern Region of England 23\% of surgeons who perform Mitchell's osteotomies used sutures to hold the osteotomies. ${ }^{7}$ In my District Hospital $90 \%$ of the hallux valgus correction was with Mitchell's osteotomy fixed with sutures, but this practice was changed after this retrospective study.

The most common late sequela of Mitchell's osteotomy is transfer metatarsalgia of the lesser toes. ${ }^{3}$ In this study $10.9 \%$ had transfer metatarsalgia, but $\mathrm{Kuo}, \mathrm{CH}$, et al., reported $20 \%$ of transfer metatarsalgia and recommend Mitchell's osteotomy as long as the indication criteria and the surgical technique are respected in a mild to moderate deformity. ${ }^{3}$ In this review even in mild and moderate cases when a suture is used for the fixation there is a high incidence of recurrence of $26.8 \%$. 
Table 3 Lesser toe procedures in feet with transfer metatarsalgia

Procedures Number

Partial Proximal phalangectomy 4

Excision of proximal interphalangeal joint 1

Pain over the bunion caused by a recurrence of the hallux valgus deformity was the main reason for the late deterioration of results, Fokter SK, et $\mathrm{al}^{11}$, all patients in this review with recurrence had pain over the bunion.

Controlling the osteotomy site in the Mitchell's procedure may be difficult and a dorsal displacement may occur, if the osteotomy is fixed with sutures. This could have lead to the high recurrence in the review.

In this study, there was not a significant change in the position of tibial sesamoid bone post-operatively in the feet with recurrence. Avascular necrosis is rare after Mitchell's osteotomies. In this review only 1.2\% had avascular necrosis but Meier PJ, et al., reported osteonecrosis in 8\% after Mitchell's osteotomies ${ }^{12}$, Desjardin, AL, et al., reported no case of avascular necrosis, pseudoarthrosis or infection. ${ }^{13}$

Recurrence of hallux valgus is a well known complication, attributable to several reasons including insufficient or no primary lateral displacement of the metatarsal head, malunion or fracture of the metatarsal osteotomy, untreated pes planovalgus, insufficient soft tissue technique or a combination of any of the above. ${ }^{14}$ In this review, none of the patients had lateral soft tissue release and all osteotomies were fixed with suture, these could be contributing factors to the recurrences, also the DMAA (distal metatarsal articular angle) was not corrected.
Of the feet with transfer metatarsalgia, five feet also had additional procedure to the lesser toes. It is a well known fact that lesser toe procedure could cause transfer metatarsalgia. (Table 3) Figures 2 and 3 are typical examples of $\mathrm{x}$-rays showing loss of correction 6 and 8 months after surgery using the Mitchell's osteotomy for bunion correction. In our department since after this review, we have stopped using sutures to hold Mitchell's osteotomies due to the high recurrence.

\section{Conclusion}

Mitchell's osteotomy can be used in the correction of mild to moderate hallux valgus but could be associated with high recurrence and transfer metatarsalgia when the osteotomy is fixed with a suture. 


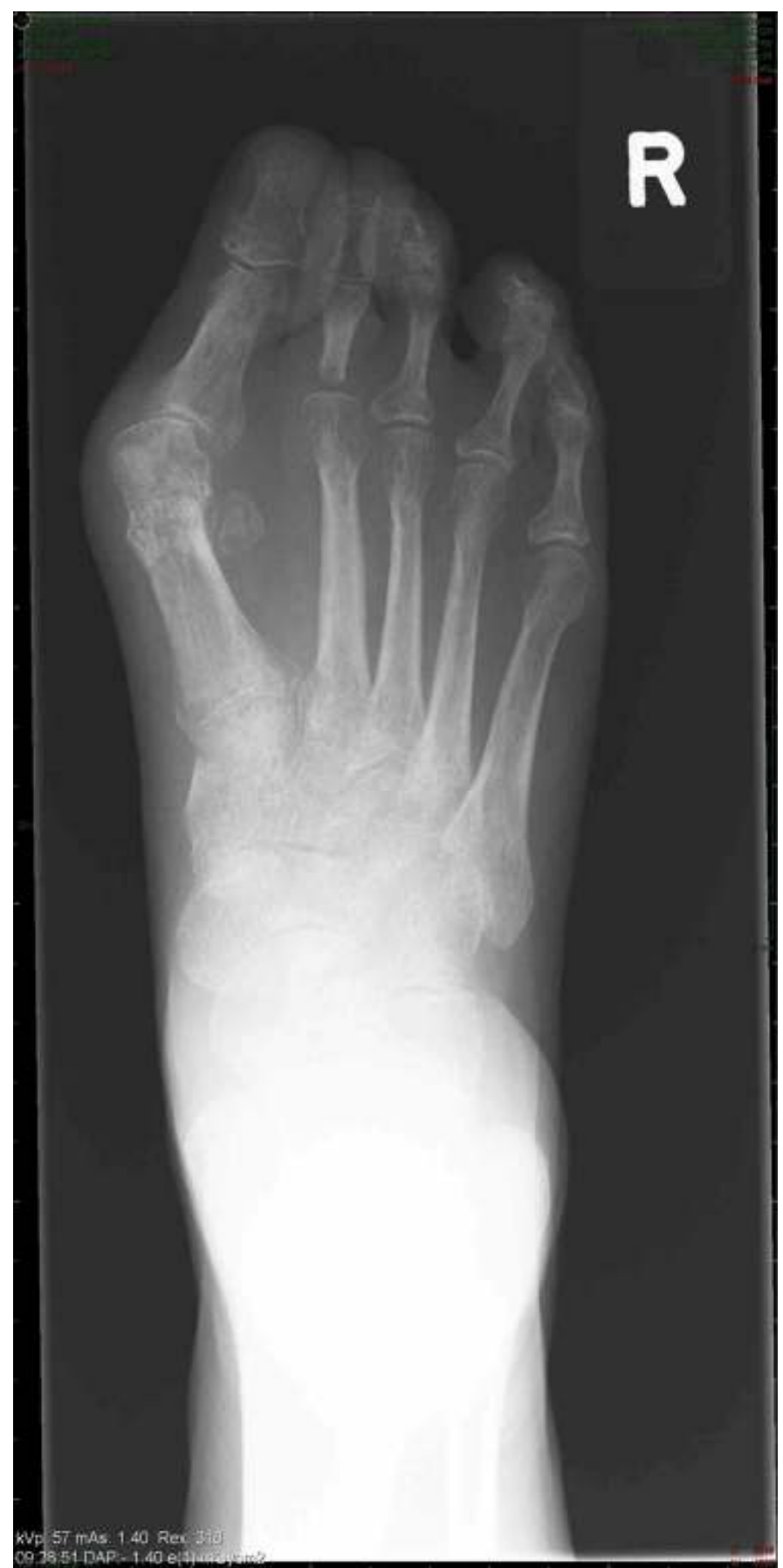

Figure 2 Example $x$-ray of lost bunion correction six weeks after the operation.

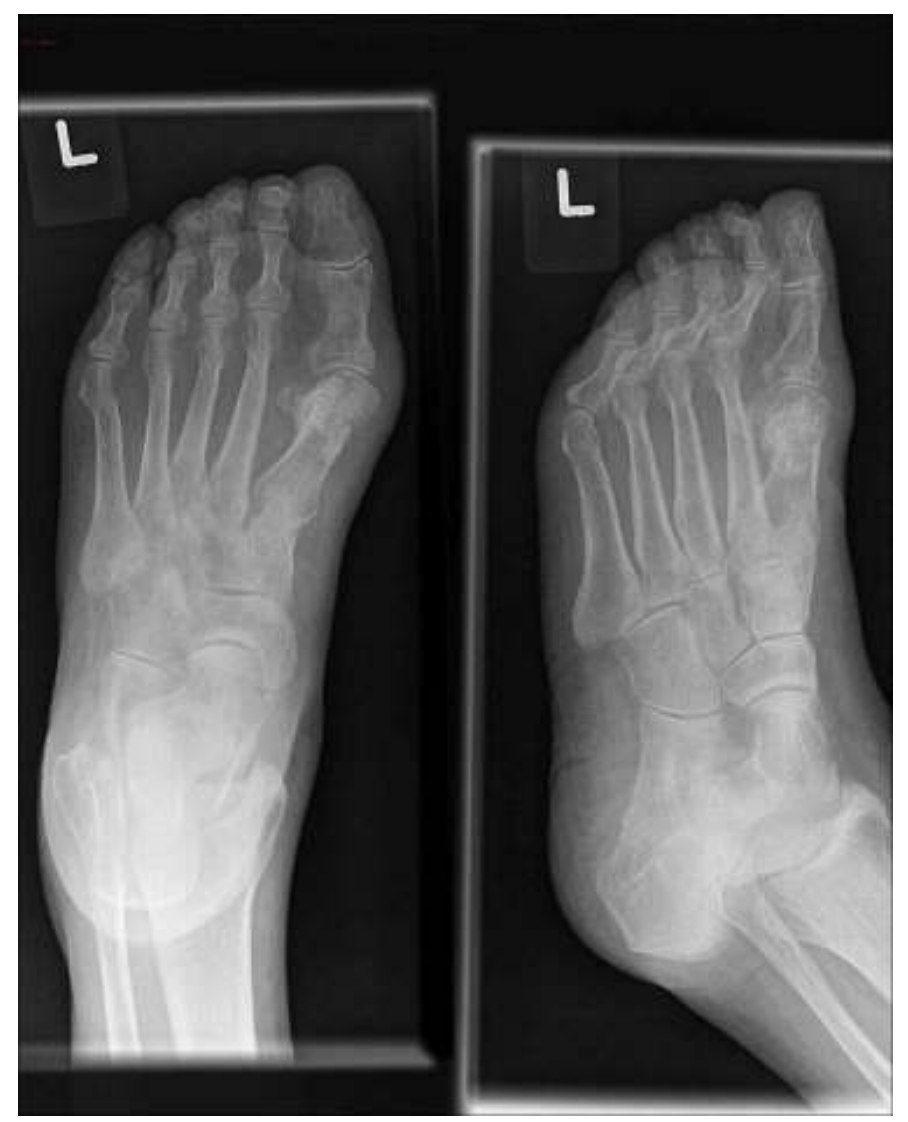

Figure 3 Example of foot x-ray eight months after surgery with healed osteotomy. There is now recurrence and transverse plane displacement of the first metatarsal.

\section{References}

1. Mitchell CL, Flemming JL, Allen R, Glenney C, Sanford GA. Osteotomy-bunionectomy for hallux valgus. JBJS 1958; 40A:4160.

2. Kelikian H. The Hallux. In: Jahss M (Ed) Disorders of the Foot. Saunders: 1982. P. 539-621.

3. Kuo CH, Huang PJ, Cheng YM, Huang KY, Chen TB, Chen YW, Lin SY. Modified mitchell osteotomy for hallux valgus . Foot \& Ankle Int 1998; 19 :(9):585-589.

4. Rossi WR, Ferreira JCA. Chevron osteotomy for hallux valgus. Foot \& Ankle Int 1992; 13:8-14,

5. Sanhudo JAV. Correction of moderate to severe hallux valgus deformity by a modified Chevron shaft osteotomy. The American Orthopaedic Foot \& Ankle society 2007.

6. Dhukaram V, Hullin MG, Kumar SC, The Mitchell and Scarf osteotomies for hallux valgus correction: a retrospective, comparative analysis using planter pressures.

J Foot Ankle Surg: 2006 45(6):400-409 
7. Gower A, Greiss ME, Briggs PJ. What is modified Mitchell's osteotomy? The Foot 2000 (10): 66-68

8. Pickering S, Zafiropoulous G, Henry APJ. Shortening and upward displacement at osteotomy of $1^{\text {st }}$ Metatarsal for Hallux Valgus. The effect on forefoot load bearing. JBJS 81B (Suppl III), 378 .

9. Kinnard P, Gordon D. A Comparison between Chevron and Mitchell osteotomies for hallux valgus.

Foot \& Ankle Int. 1984 4(5):241-243

10. David-West KS, Moir JS, Radiological assessment of tibial sesamoid position after Scarf Osteotomy for Hallux valgus correction. Foot and Ankle Surgery 2002; 8: 209-212.

11. Fokter SK, Podobnik J, Vengust V. Late results of modified Mitchell's procedure for the treatment of hallux valgus. Foot \& Ankle Int. 1999 May; 20(5):296-300

12. Meier PJ, Kenzora JE. The risks and benefits of first metatarsal osteotomies. Foot \& Ankle Int 1985; Aug: (1):7-17

13. Desjardins, AL, Hajj C, Racine L, Fallaha M, Bornais S.

Mitchell's osteotomy in the treatment of hallux valgus. Ann Chir 1993; 47(9):894-899.

14. Coughlin MJ, Mann RA, Saltzman CL (eds). Surgery of the Foot and Ankle. Edition 8, Part 1 Chapter 6: Hallux valgus.

Philadelphia, PA: Mosby Elsevier; 2007:283-362. 